\title{
Mitigating the Table-Overflow Attack in Software-Defined Networking
}

\author{
Tong Xu, Deyun Gao ${ }^{(}$, Ping Dong, Chuan Heng Foh, Senior Member, IEEE, and Hongke Zhang
}

\begin{abstract}
Software-defined networking (SDN) is a promising 2 network paradigm for future Internet. The centralized con3 troller and simplified switches replace the traditional complex 4 forwarding devices, and make network management convenient. 5 However, the switches in SDN currently have limited ternary 6 content addressable memory to store specific routing rules from 7 the controller. This bottleneck provokes cyber attacks to over\& load the switches. Despite existing some countermeasures for 9 such attacks, they are proposed based on simplified attack pat10 terns. In this paper, we review the table-overflow attack using a 11 sophisticated attack pattern. In the attack pattern, attack flows 12 are targeted at their middle hops instead of endpoints. We first 13 define potential targets in the network topology, then we pro14 pose three specific traffic features and a monitoring mechanism 15 to detect and locate the attackers. Further, we propose a mitiga16 tion mechanism to limit the attack rate using the token bucket 17 model. With the control of token add rate and bucket capac18 ity, it avoids the table overflow on the victim switch. Extensive 19 simulations in different types of topologies and experiments 20 in our testbed are provided to show the performance of our 21 proposal.
\end{abstract}

Index Terms-Software-defined networking, OpenFlow, comгз munication system security, table-overflow attack.

\section{INTRODUCTION}

${ }_{25} \mathrm{O}$ OFTWARE-DEFINED Networking (SDN) is considered ${ }_{26} \bigcirc$ as one promising next generation network architecture ${ }_{27}$ because of its high flexibility in network management. Many 28 companies, such as Google and Amazon, have deployed SDN 29 devices in their backbone and data center [1], [2]. The key so concept of SDN lies in the decoupling of the data plane and 31 the control plane [3]. In SDN, the switches are free from rigid 32 routing computation and can be focused on packet forwarding. ${ }_{33}$ The centralized controller now is in charge of the routing rule ${ }_{34}$ computation and assignment.

Manuscript received March 15, 2017; revised July 21, 2017 and September 20, 2017; accepted September 27, 2017. This work was supported by NSFC under Grant No. 61232017, 61272504, the 973 Program under Grant No. 2013CB329100 and the Fundamental Research Funds for Central Universities under Grant No. 2016YJS018. The associate editor coordinating the review of this paper and approving it for publication was R. Riggio. (Corresponding author: Deyun Gao.)

T. Xu, D. Gao, P. Dong, and H. Zhang are with the National Engineering Laboratory for Next Generation Internet Interconnection Devices, School of Electronic and Information Engineering, Beijing Jiaotong University, Beijing 100044, China (e-mail: 14111037@bjtu.edu.cn; gaody@bjtu.edu.cn; pdong@bjtu.edu.cn; hkzhang@bjtu.edu.cn).

C. H. Foh is with 5GIC, Institute for Communication Systems, Department of Electrical and Electronic Engineering, University of Surrey, Surrey GU1 2UX, U.K. (e-mail: c.foh@surrey.ac.uk).

Digital Object Identifier 10.1109/TNSM.2017.2758796
Since the switches become highly primitive, the assigned routing rules from the controller are expected to be precise. Currently, OpenFlow [4] is the most widely used control link protocol owing to its fine-grained routing rules (i.e., flow entries) and abundant flow statistics. To support OpenFlow protocol better, the switches have to use ternary content addressable memory (TCAM) to store flow entries. However, considering the high cost and highpower consumption [5], the current OpenFlow-enabled switches usually have limited flow table, which supports a few thousand flow entries [6], [7]. For example, without the external TCAM, the NoviSwitch 2150 [8] can only store 16,384 flow entries.

As a result, how to use such limited cache efficiently and safely has been an active field of research. To help the switches store more flow entries without adding TCAMs, several proposals attempt to split the workload between the switches [9], [10] or aggregate redundant flow entries [11], [12]. Although these proposals are useful for normal users, they could be inefficient when attackers attempt to exhaust the switches. We call such attacks the table-overflow attack in this paper. Many recent studies [13]-[15] have paid attention to the table-over flow attack due to its significant negative impact, including higher energy consumption, flow table overflow and normal communication breakdown.

While the awareness of the table-overflow attack has been raised, existing studies [13]-[17], [32] mainly consider the attack with simplified attack patterns. In the simplified attack patterns, only one or a few attackers attempt to overload a target switch using lots of unmatched attack flows. Moreover, the table-overflow attackers are assumed to be brute-force. It means that, the attackers choose their common ingress or egress (physically attached) switch as the target. Based on the simplified cases, a few security suggestions and countermeasures [18], [32] are proposed in the literature.

However, in the real world operation, a sophisticated attack pattern is more attractive to the cyber attackers. In the sophisticated attack pattern, a group of attackers launch the table-overflow attack to a same target switch. And the target switch is a middle hop switch instead of their physically attached switches. Since when attackers launch the tableoverflow attack to their physically attached switch, they risk exposing themselves. Besides, the flow table consumption on the target switch (we call these consumption the attack pay- 7 ment) causes the same amount of flow table consumption on 79 their physically attached switches (we call these consumption 80 the attack cost). Therefore, the table-overflow attack is more ${ }_{81}$ 
82 likely to be launched by a group of attackers with the pur83 pose of splitting the attack cost on their physically attached 84 switches and hiding themselves.

85 In the sophisticated attack pattern, the attackers tend to 86 choose a hot switch which has fewer vacancies in the flow ${ }_{87}$ table as the target switch. Their attack flows use the target 88 switch as the middle hop instead of the endpoints, because 89 when lots of abnormal flows share the same traffic feature, 90 such as the destination IP, these flows trigger an alarm in 91 the existing security mechanisms [18], [19]. To identify a hot 92 switch, an attacker may seek to use some common social ${ }_{93}$ engineering attacks to the network manager, or use route 94 trace tools, or even invade the central controller to obtain the 95 network topology to locate a hot switch.

96 Knowing the basic characteristics of the sophisticated table${ }_{97}$ overflow attack, the objective of our work is to defend against 98 it in SDN. Given an SDN topology, we first build a mathe99 matical model to formulate the flow table consumption and 100 the attack. Based on it, we define the potential targets. Then 101 we propose three traffic features and a monitoring mechanism 102 to detect the table-overflow attack and locate the attackers. ${ }_{103}$ Further, we propose a mitigation mechanism to limit the rate 104 of attack flows using the token bucket model. It controls the 105 token add rate and the bucket capacity with the consideration 106 of the current flow table vacancy on the victim switch. Both 107 the monitoring and mitigation mechanisms are embedded into 108 the original routing process of SDN. Extensive simulations 109 and experiments are conducted to evaluate our proposal. Here 110 we summarize the main contributions of this paper.

111 - Most of the related studies investigate the table-overflow 112 attack using simplified attack patterns. In this work, we ${ }_{113}$ consider a sophisticated attack pattern and propose a 114 countermeasure to the sophisticated table-overflow attack.

115 - We build a practical mathematical model to formulate the 116 flow table consumption and the attack. We provide a way 117 to define the potential targets of the table-overflow attack. 118 - Three specific traffic features and a monitoring mech119 anism are proposed during the original routing process 120 of SDN. It detects the attack and locates the attacker 121 attached switches.

122 - A mitigation mechanism is proposed using the token ${ }_{123}$ bucket model. Given the current flow table vacancy on 124 the victim switch, it limits the rate of attack flows and 125 avoids the table overflow under different attack rates.

126 The rest of the paper is organized as follows. Some related 127 work is introduced in Section II. The basic concept of SDN 128 and the table-overflow attack is provided in Section III. In ${ }_{129}$ Section IV, we build the mathematical model to formulate ${ }_{130}$ the flow table consumption and the table-overflow attack. ${ }_{131}$ The monitoring mechanism and the mitigation mechanism are 132 proposed in Sections V and VI, respectively. In Section VII, ${ }_{133}$ we show the simulation and experiment results of our proposal. ${ }_{134}$ Finally, we conclude this paper in Section VIII.

\section{5 \\ II. RELATED WORK}

${ }_{136}$ In this section, we introduce some related work about the ${ }_{137}$ table overflow problem of SDN.

\section{A. Table Overflow Avoidance Under Normal Circumstances ${ }_{138}$}

For SDN-enabled switch vendors, such as NoviFlow [8] and ${ }_{139}$ Pica8 [20], the only mature way to store more flow entries 140 seems to be adding the number of TCAMs. In the litera- ${ }_{141}$ ture, to find a better solution, the existing studies mainly go 142 along two directions, i.e., heterogeneous flow entry and cache ${ }_{143}$ optimization.

144

The key concept of heterogeneous flow entry approaches is 145 that not all arrived flows need an individual wildcard flow entry 146 stored in TCAM. For example, DevoFlow [21] classifies flows 147 into two types: the transient mice flow and the persistent ele- 148 phant flow. It clones the mice flow's packet header to create an 149 exact-match flow entry. Then the exact-match flow entry can 150 be moved from TCAM to RAM. Similarly, Nguyen et al. [22] ${ }_{151}$ only assign specific rules to the elephant flows, the mice flows 152 are handled by the default rules in switches. CacheFlow [23] 153 only stores the most popular rules in a small TCAM. The rest ${ }_{154}$ of rules are cached in software switches using RAM. The rules 155 stored in TCAM have a higher priority than the rules stored 156 in RAM.

157

Besides, Banerjee and Kannan [24] propose Tag-In-Tag 158 based on their former study about TCAM [5]. They utilize ${ }_{159}$ a combination of path tag and flow tag (24 bits in total) 160 instead of the original flow entry (356 bits in total) to match 161 a flow. The path tag exploits the path similarity between dif- 162 ferent flows. Likely, Huang et al. [25] divide flow entries into 163 the universal rules and the local rules. They conduct different 164 partition and allocation algorithms for different kinds of flow 165 entries. In this way, these proposals reduce redundant rules in 166 switches. 167

In contrast, the key concept of cache optimization 168 approaches is that not all cached flow entries in TCAM need 169 to follow a same and rigid update mechanism. For example, 170 SmartTime [26] sets the idle timeout dynamically according to 171 its observation of the repeated flow and the real time TCAM 172 occupation. It aims to improve the utilization of TCAM (i.e., 173 use a smaller idle timeout) while ensuring that TCAM miss 174 does not increase (i.e., fewer flows are repeated). Similarly, 175 MWFP [27] adjusts the idle timeout of flows with the con- 176 sideration of the average interval between packets. It reduces 177 the TCAM updates by setting a bigger idle timeout for the 178 long-distance and unstable flow. 179

Besides, FDRC [28] aims at fine-grained cache manage- 180 ment. It sets a timer for each flow entry to estimate the time ${ }_{181}$ of next hit. The rarely hit rules have a larger timer value and ${ }_{182}$ will be evicted once TCAM is full. Likely, MMS [29] cleans ${ }_{183}$ the rarely used or obsolete flow entries. It resides in the SDN ${ }_{184}$ controller and monitors the activity of applications. To free ${ }_{185}$ TCAM in the switches, MMS evicts the flow entries once 186 their applications in the controller are terminated. $\quad{ }_{187}$

\section{B. Table Overflow Avoidance Under DoS Attacks}

188

The aforementioned proposals improve the accommodation 189 of flow entries in SDN, however they are inefficient to handle 190 malicious flows from cyber attackers. It has been proven that 191 the limited flow table space can be easily exhausted by table- 192 overflow attackers [16], [17]. To deal with such security issues, 193 several mechanisms are proposed recently. 


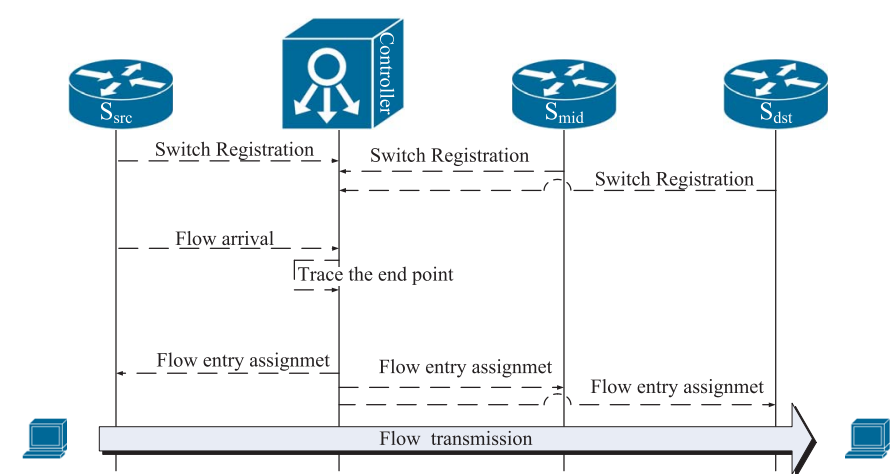

Fig. 1. SDN's routing process: global network view establishment, routing rule calculation and routing rule assignment.

195 AVANT-GUARD [30] is a security mechanism embedded in 196 the SDN-enabled switches. It consists of a connection migra197 tion module and an actuating trigger module. The connection 198 migration module is designed to monitor TCP session fail199 ures. The actuating trigger module is used to activate several 200 predefined routing rules when lots of TCP session failures are 201 observed. In this way, it protects the controller and switches 202 from the SYN flooding attack.

203 Dong et al. [31] propose a detection method for the DDoS 204 attack to the controller and switches. Such a DDoS attack uti205 lizes a large number of low-traffic flows to exhaust SDN's rout206 ing system. The low-traffic flows have fewer packets than the 207 normal flows, and they can lead to significant resources con208 sumption in both the data plane and the control plane. In the 209 proposal, the authors use SPRT (Sequential Probability Ration 210 Test) to control the false negative and false positive rates.

211 The most related work to this paper is the QoS-aware mit212 igation strategy [32]. It prepares the idle flow table resources 213 in the data plane for the table-overflow attack. By redirecting 214 attack flows from saturated switches to idle switches, it makes 215 SDN more resilience to such an attack. However, since the 216 redirection action cannot limit the attack rate, its performance 217 relies on the amount of idle flow table resources, and it may 218 be unsatisfactory when there are no adequate idle switches 219 or the attack rate is high. Moreover, the effectiveness of this 220 approach relies on the identification of the attack. Since the 221 authors did not provide a proper attack monitoring method, the 222 effectiveness of their proposed solution is somewhat limited.

\section{III. PRELIMINARY KNOWLEDGE}

224 In this section, we introduce the original routing process of 225 OpenFlow-based SDN and the table-overflow attack.

\section{A. The Routing Process of OpenFlow-Based SDN}

227 According to OpenFlow protocol, the SDN-enabled switch 228 has to register its configuration and status before it can forward 229 packets in the network. During this process, the controller 230 builds a topology of the entire network. As shown in Fig. 1, 231 when an unmatched flow arrives at $S_{s r c}, S_{s r c}$ sends a request ${ }_{232}$ to the controller. Then the controller traces the endpoint $S_{d s t}$ 233 and finds the middle hop $S_{\text {mid }}$ in the established topology. 234 To make sure that the arrived flow is continuously transmitted, 235 all switches in the routing path have to install a flow entry.
TABLE I

The Capacities of Most Used Switches

\begin{tabular}{l|l}
\hline \hline Context & Capacity \\
\hline Commercial switches [6, 20] & $2,000 \sim 8,000$ \\
OpenvSwitch [33] & $15,000 \sim 20,000$ \\
NoviSwitch 2150 [8] & 16,384 \\
NoviSwitch 2128, 2122, 1248 [8] & $1,000,000$ \\
Simulation settings in related studies [16, 32] & $1,500 \sim 8,000$ \\
\hline
\end{tabular}

During this routing process, the new arrived flow not only 236 leads to flow entry installations on its ingress switch $S_{S r C}{ }_{237}$ and egress switch $S_{d s t}$, but also on the middle hop (logically 238 attached) switch $S_{\text {mid }}$.

\section{B. The Table-Overflow Attack in SDN}

Currently, the switches in OpenFlow-based SDN usually 241 have limited cache for flow entries. Table I shows the cache 242 sizes of different OpenFlow-enabled switches. We can see that 243 the OpenFlow-enabled switch generally can hold a few thou- 244 sands of flow entries. Such bottleneck provokes cyber attackers 245 to overload the switches. By generating lots of unmatched 246 flows in a short time, the attackers trick the controller into 247 assigning flow entries to a target switch intensively. Once the 248 cache of the target switch is full of these flow entries, the tar- 249 get switch will be paralysed and the legitimated flows will be 250 delayed or even dropped.

251

We note that the NoviSwitch 2128, 2122 and 1248 can 252 support 1 million of flow entries using large TCAMs. Such 253 amount of cache definitely can withstand most of the table- 254 overflow attack in the simplified model [13]-[17], [32]. In the 255 simplified attack model, the attack flows use the target switch as 256 their ingress or egress in their routing paths, e.g., $S_{s r c}$ or $S_{S r c}$ in 257 Fig. 1. Such attack flows can be easily aggregated by the target 258 switch according to their common ingress or egress. Unless 259 the brute-force attacker targets a switch with fewer vacancies 260 in the cache, the attacker has to generate attack flows at a very 261 high rate.

262

However, in the sophisticated attack model, the attack flows 263 use the target switch as their middle hop in their routing paths, 264 e.g., $S_{\text {mid }}$ in Fig. 1. Using different paths with a same middle 265 hop, the attackers can accumulate useless flow entries on the 266 middle hop. In the real world operation, the switch serves as 267 a middle hop frequently in the network. This makes lots of 268 available routing paths for the attackers. More importantly, 269 such attack flows are difficult to be aggregated according to 270 their common middle hop. To overflow a switch using large 271 TCAMs, the sophisticated attack can utilize more routing paths 272 and share the attack cost accordingly.

273

Besides, adding the number of TCAMs in the switch is an 274 efficient way to improve the reliability of switch but not secu- 275 rity. Especially for the sophisticated table-overflow attackers, 276 adding cache may provoke more intense attacks. Therefore, 277 the motivation of this paper is to keep the limited or finite 278 cache from the sophisticated attackers.

279

IV. System Model And Problem Description 280

In this section, we build a mathematical model to formu- 281 late the above mentioned table-overflow attack. When switches 282 


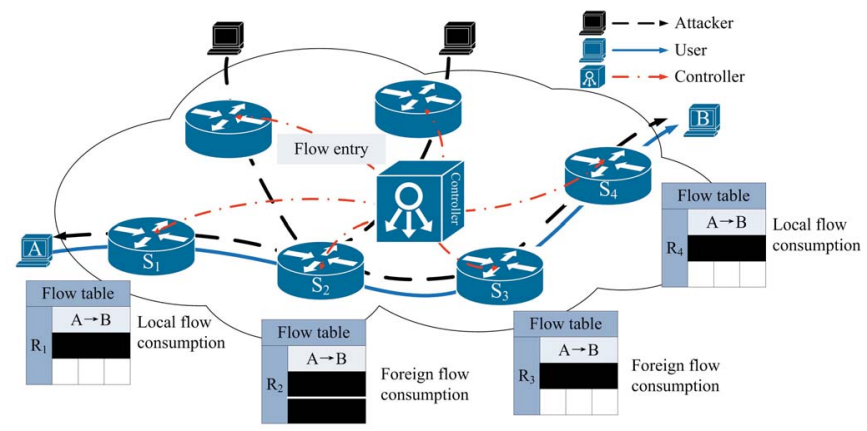

Fig. 2. Classification of the flow table consumption.

283 finish their registration, the global view in the network con284 troller can be viewed as an undirected and unweighted graph ${ }_{285} G=(S, L)$, where $S$ represents the switch set and $L$ is 286 the physical link set. We use $N$ to denote the number of 287 switches. Then we formulate the flow table consumption in 288 a discrete time model, where the time horizon consists of 289 a series of time slots. We use $l$ to denote the length of the 290 time slot.

291 During a time slot $t$, the flow entries assigned to $S_{i}$ are 292 viewed as a set $R_{i}^{t}$. Then we define the flow table consumption 293 of $S_{i}$ in time slot $t$ as

$$
C_{i}(t)=\operatorname{card}\left(R_{i}^{t}\right),
$$

295 where $\operatorname{card}\left(R_{i}^{t}\right)$ is the cardinality of set $R_{i}^{t}$. To give a further 296 view of $C_{i}(t)$, we divide $R_{i}^{t}$ into two parts: the local flow used 297 set $R_{i}^{t, L}$ and the foreign flow used set $R_{i}^{t, F}$. The local flow of ${ }_{298} S_{i}$ uses $S_{i}$ as the ingress or egress switch, the foreign flow of $299 S_{i}$ uses $S_{i}$ as the middle hop. For example, as shown in Fig. 2, 300 the users' flow is a local flow for $S_{1}$ and $S_{4}$, and it is a foreign 301 flow for $S_{2}$ and $S_{3}$. It is obvious that $R_{i}^{t, L}$ and $R_{i}^{t, F}$ have the 302 following properties:

303

$$
\left\{\begin{array}{l}
R_{i}^{t, L} \cup R_{i}^{t, F}=R_{i}^{t} \\
R_{i}^{t, L} \cap R_{i}^{t, F}=\varnothing
\end{array}\right.
$$

304 Then we define the local flow consumption (i.e., $\left.C_{i}^{L}(t)\right)$ and 305 the foreign flow consumption (i.e., $\left.C_{i}^{F}(t)\right)$ as

$$
\left\{\begin{array}{l}
C_{i}^{L}(t)=\operatorname{card}\left(R_{i}^{t, L}\right) \\
C_{i}^{F}(t)=\operatorname{card}\left(R_{i}^{t, F}\right) .
\end{array}\right.
$$

307 Therefore, the flow table consumption $C_{i}(t)$ is

308

$$
C_{i}(t)=C_{i}^{L}(t)+C_{i}^{F}(t) .
$$

309 We use $\left\langle S_{i} \cdot S_{j}\right\rangle$ to denote the routing path between $S_{i}$ and $S_{j}$ 310 (flows from $S_{i}$ to $S_{i}$ are not considered in this paper). For the 311 normal flows on the path, we formulate them as the generally 312 used Poisson process [32]. Considering the fact that different ${ }_{313}$ paths have different amount of traffic. We use $\lambda_{i \cdot j}$ to denote 314 the unmatched flow arrival rate of path $\left\langle S_{i} \cdot S_{j}\right\rangle$ and set it 315 randomly for different paths. All symbols and variables used 316 in this paper are summarized in Table II.

317 When the table-overflow attackers choose $S_{i}$ as the target 318 switch, they generate flows on different paths which include ${ }_{319} S_{i}$ as the middle hop. These attack flows lead to a huge foreign

\begin{tabular}{|c|c|}
\hline Notations & Description \\
\hline$G$ & $\begin{array}{l}\text { Undirected and unweight graph, } G=(S, L) . S \text { is the SDN- } \\
\text { enabled switch set, } S=\left\{S_{i} \mid i=1,2, \ldots N\right\}, L \text { is the } \\
\text { physical link set }\end{array}$ \\
\hline $\operatorname{card}(A)$ & The cardinality of set A, e.g. $\operatorname{card}(\{1,2,3,5\})=4$ \\
\hline $\mathbb{P}$ & The probability \\
\hline $\mathbb{E}$ & The mathematical expectation \\
\hline$D_{i}$ & Degree of $S_{i}$ \\
\hline$d_{i}$ & $\begin{array}{l}\text { Virtual degree of } S_{i} \text {, i.e. the number of paths which include } \\
S_{i} \text { as the middle hop }\end{array}$ \\
\hline$l$ & Length of each time slot, e.g. time slot t \\
\hline$R_{i}^{t}$ & Set of flow entries assigned to $S_{i}$ during time slot t \\
\hline$R_{i}^{t, L}$ & Flow entries for local flows in $R_{i}^{t}$ \\
\hline$R_{i}^{t, F}$ & Flow entries for foreign flows in $R_{i}^{t}$ \\
\hline$C_{i}^{2}(t)$ & Flow table consumption of $S_{i}$ in time slot $\mathrm{t}$ \\
\hline$C_{i}^{L}(t)$ & Local flow consumption of $S_{i}$ in time slot t \\
\hline$C_{i}^{F}(t)$ & Foreign flow consumption of $S_{i}$ in time slot t \\
\hline$\left\langle S_{i} \cdot S_{j}\right\rangle$ & The routing path between $S_{i}$ and $S_{j}$ \\
\hline$\lambda_{i, j}$ & Flow arrival rate of path $\left\langle S_{i} \cdot S_{j}\right\rangle$ \\
\hline$\lambda_{p}$ & $\begin{array}{l}\text { Flow arrival rate of path }\left\langle S_{i} \cdot S_{j}\right\rangle \text { in the simplified traffic } \\
\text { model }\end{array}$ \\
\hline$\lambda_{i}^{F, j}$ & $\begin{array}{l}\text { The arrival rate of flows which use } S_{i} \text { as the middle hop and } \\
S_{i} \text { as the ingress or egress }\end{array}$ \\
\hline$\lambda_{i}^{F}$ & Foreign flow arrival rate of $S_{i}, \lambda_{i}^{F}=\sum_{S_{j} \in S, j \neq i} \lambda_{i}^{F, j}$ \\
\hline$\lambda_{i}^{L}$ & Local flow arrival rate of $S_{i}, \lambda_{i}^{L}=\sum_{S_{j} \in S, j \neq i} \lambda_{i, j}$ \\
\hline $\begin{array}{l}\lambda_{i} \\
\lambda_{G}\end{array}$ & $\begin{array}{l}\text { Flow arrival rate of } S_{i}, \lambda_{i}=\lambda_{i}^{F}+\lambda_{i}^{L} \\
\text { Flow arrival rate of } G, \lambda_{G}=\sum \lambda_{i, j}\end{array}$ \\
\hline
\end{tabular}
320 flow consumption $C_{i}^{F}(t)$ during the attack.
TABLE II

VARIABLES AND NOTIONS

However, not all switches are attractive for attackers. As ${ }_{321}$ shown in Fig. 2, the instinct of the attackers makes them ${ }_{322}$ choose a hot switch (e.g., $S_{2}$ ) instead of $S_{1}$ as the target, since ${ }_{323}$ $S_{2}$ is responsible for more normal flows and has fewer vacan- ${ }_{324}$ cies than $S_{1}$. Therefore, before we monitor and further mitigate ${ }_{325}$ the table-overflow attack, we should identify the potential ${ }_{326}$ targets in a given graph $G$.

\section{Monitoring the TABle-Overflow Attack 328}

In this section, we first define the potential targets according ${ }_{329}$ to their common feature. Then we design three traffic features 330 to monitor the attack for the potential targets. Finally, we show ${ }_{331}$ how our monitoring mechanism is embedded into the routing ${ }_{332}$ process of SDN.

\section{A. Definition of Potential Targets}

334

To find potential targets in a given graph $G$, we can first 335 exclude some switches that are secure from the table-overflow 336 attack. Since the attackers generate attack flows on different ${ }_{337}$ paths which include the target as the middle hop. Switches ${ }_{338}$ that only act as the ingress or egress are not the candidate ${ }_{339}$ of potential target. For example, as the graph $G$ shown in 340 Fig. 3(a), $S_{1}, S_{4}$ and $S_{5}$ are not middle hops in any routing 341 paths in $G$, and their foreign flow consumption $C_{i}^{F}(t) \equiv 0$ since 342 their degree $D_{i}=1$. Therefore, we select potential targets from ${ }_{343}$ switches which satisfy $D_{i}>1$.

We use Algorithm 1 to build virtual graphs for switches with 345 $D_{i}>1$. As shown in Fig. 3(b) and Fig. 3(c), each virtual graph 346 consists of a central switch $S_{i}$ and several virtual neighbors. ${ }_{347}$ The virtual neighbors are the paths in graph $G$, which include ${ }_{348}$ the central switch as the middle hop. When flows traverse in 349 


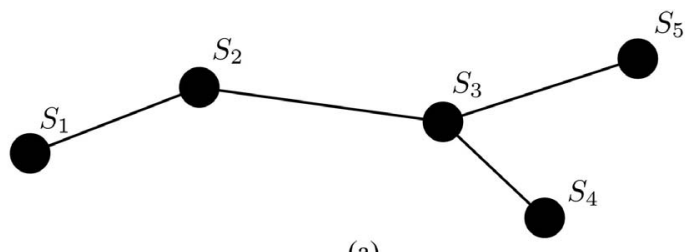

(a)

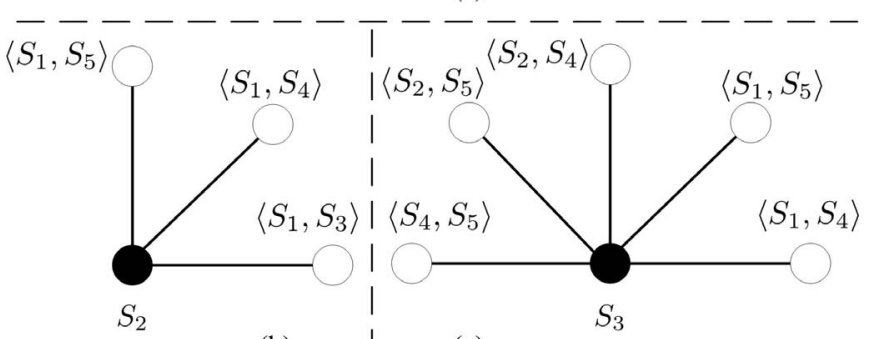

(b)

(c)

Fig. 3. An example of building virtual graphs using $G$, in the virtual graphs, the paths in $G$ represent virtual neighbors.

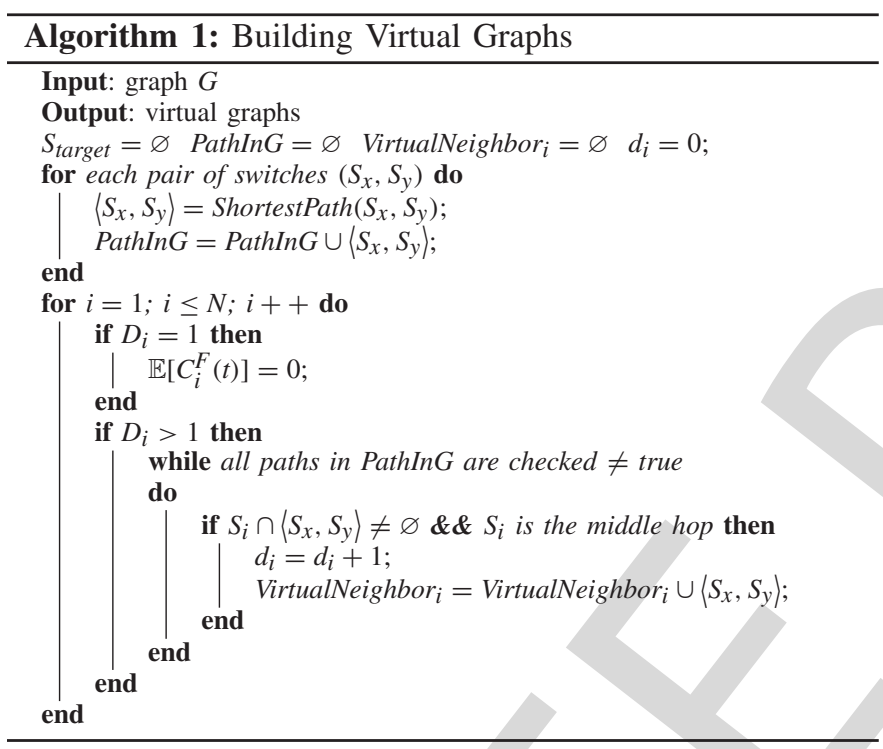

350 graph $G$, the controller finds the shortest (in terms of hop, 351 delay or some commercial cost) path between its ingress and 352 egress switches. In this paper, the least number of hops is the 353 shortest path. Then we use $d_{i}$ to denote the virtual degree of $S_{i}$, 354 and the virtual degrees of $S_{2}$ and $S_{3}$ are 3 and 5, respectively. 355 Then the question becomes whether we can simply treat all 356 switches with $D_{i}>1$ as the potential targets in $G$. For small 357 scale graphs, this definition may be workable since there are 358 a few switches in the topology. While for large scale graphs 359 this is not sufficient and can lead to lots of potential targets. 360 Meanwhile, it should be note that, the virtual neighbors of the 361 switch with small $d_{i}$ may also belong to the switch with large $362 d_{i}$. For example, in Fig. 3(b) and Fig. 3(c), two thirds of the ${ }_{363} S_{2}$ 's virtual neighbors are also belong to $S_{3}$. It indicates that, 364 for large scale graphs, even attackers try to overload a switch 365 with small $d_{i}$, some switches with large $d_{i}$ are also involved 366 into the attack. And due to the fact that switches with large $d_{i}$ 367 usually has fewer flow table vacancy than the ones with small ${ }_{368} d_{i}$, switches with large $d_{i}$ will be overloaded first.

369 It's obviously hard to draw a clear line between switches 370 with large $d_{i}$ and small $d_{i}$, and make sure switches with small $d_{i}$ will not exhausted before the rest under any rates of table- 371 overflow attacks.

Therefore, we narrow the range of potential targets with the 373 consideration of a simplified traffic model in graph $G$. In this 374 simplified traffic model, each path has the same flow arrival 375 rate $\lambda_{p}$. It should be noted that we do not use this model to 376 monitor the table-overflow attack, what we do is to provide a 377 possible way to narrow the range of potential targets in graph 378 $G$. Then the expected local flow consumption during a single 379 time slot is

$$
\mathbb{E}\left[C_{i}^{L}(t)\right]=(N-1) \lambda_{p} l .
$$

And the expected foreign flow consumption during a single 382 time slot is

$$
\mathbb{E}\left[C_{i}^{F}(t)\right]= \begin{cases}0, & D_{i}=1 \\ d_{i} \lambda_{p} l, & D_{i}>1\end{cases}
$$

according to (3). To define the potential targets, we use the 385 foreign-local ratio as following,

$$
\frac{\mathbb{E}\left[C_{i}^{F}(t)\right]}{\mathbb{E}\left[C_{i}^{L}(t)\right]}=\frac{d_{i} \lambda_{p} l}{(N-1) \lambda_{p} l}=\frac{d_{i}}{N-1} .
$$

It reflects the topology feature of $S_{i}$ in graph $G$. When the 388 foreign-local ratio is bigger than 1 , i.e., $d_{i}>(N-1)$, switch 389 $S_{i}$ has more paths for its foreign flows than its local flows, 390 and it may have less flow table space than the rest switches. 391 In this paper, we denote the switch with $d_{i}>(N-1)$ as the 392 potential target. For example, as shown in Fig. 3, $S_{3}$ is the 393 potential target while the rest switches are not. As discussed, 394 its hard to draw a clear line between switches with large $d_{i}$ and 395 small $d_{i}$, we do not indicate that switches with $d_{i} \leq(N-1) 396$ (like $S_{2}$ in Fig. 3) are free from the table-overflow attack. 397 In contrast, we believe that some of the excluded switches 398 can be added as the potential targets by other definitions with 399 different viewpoints. And as for the numerous switches with 400 $d_{i} \leq(N-1)$, the passive detection method [34] can be a more 401 proper way to monitor the table-overflow attack.

402

\section{B. Traffic Features}

403

Knowing the potential targets, we propose the following 404 traffic features to monitor the attack: growth of foreign flow 405 consumption $(G F F C)$, deviation of flow amount $(D F A)$ and 406 commonness of flow entry $(C F E)$.

1) Growth of Foreign Flow Consumption (GFFC): 408 Although the attackers can hide themselves by splitting the 409 attack cost, to exhaust the flow table in the target switch $S_{i}$, the ${ }_{410}$ attack payment $C_{i}^{F}(t)$ must be noteworthy. Therefore, GFFC ${ }_{411}$ is defined as

$$
\operatorname{GFFC}_{i}(t)=\frac{C_{i}^{F}(t)}{l}=\frac{\operatorname{card}\left(R_{i}^{t, F}\right)}{l} .
$$

During the table-overflow attack, $G F F C_{i}(t)$ increases linearly 414 with the attack rate. A larger $G F F C_{i}(t)$ indicates a burst of 415 foreign flow consumption in $S_{i}$.

416

2) Deviation of Flow Amount (DFA): The burst of foreign 417 flow consumption may also be caused by a sudden increase of 418 normal flows. In order to differentiate the table-overflow attack 419 
420 from the normal flow bust, we propose $D F A$. The burst normal 421 flows are usually related to special events, such as popular 422 live matches and sudden news. These flows from distributed 423 users rarely share the same middle hop in the network. In 424 contrast, the attack flows have a strong directionality, they are 425 clustered at the $d_{i}$ paths of the target switch. Therefore, DFA 426 is defined as

$$
{ }_{427} D F A_{i}(t)=\frac{\mathbb{P}\left\{F A_{d_{i}}=\operatorname{card}\left(R_{i}^{t, F}\right)\right\}}{\mathbb{P}\left\{F A_{G}=\frac{1}{2} \sum_{i=1}^{N} \operatorname{card}\left(R_{i}^{t, L}\right)\right\}},
$$

428 where variable $F A_{d_{i}}$ and variable $F A_{G}$ represent the arrived 429 flow amount of the $d_{i}$ paths and the whole network in time 430 slot $t$, respectively. We use $\lambda_{i}^{F}$ and $\lambda_{G}$ to denote the flow arrival ${ }_{431}$ rate of the $d_{i}$ paths and the whole network, then variable $F A_{d_{i}}$ ${ }_{432}$ follows Poisson $\left(\lambda_{i}^{F} l\right)$ and variable $F A_{G}$ follows Poisson $\left(\lambda_{G} l\right)$. ${ }_{433}$ During the table-overflow attack, all the burst attack flows ${ }_{434}$ are clustered at the $d_{i}$ paths, and they make $D F A_{i}(t)$ decease 435 greatly. During the normal flow burst, only part of the burst 436 flows are accidentally clustered at the $d_{i}$ paths, and they make ${ }_{437} D F A_{i}(t)$ change slightly.

438 3) Commonness of Flow Entry $(\mathrm{CFE})$ : To locate the attack439 ers, we measure the switches presented in the virtual graph 440 using $C F E$. When $S_{j}$ is the attacker attached switch and $S_{i}$ is ${ }_{441}$ the target, the number of common flow entries between $R_{j}^{t, L}$ 442 and $R_{i}^{t, F}$ increases greatly. Therefore, $C F E$ is defined as

$$
{ }_{443} C_{F}(t)=\frac{\operatorname{card}\left(R_{j}^{t, L} \cap R_{i}^{t, F}\right)}{\lambda_{i}^{F, j} l},
$$

${ }_{444}$ where $R_{j}^{t, L} \cap R_{i}^{t, F}$ represents the common flow entries between ${ }_{445} S_{i}$ and $S_{j}$. We use $\lambda_{i}^{F, j}$ to denote the arrival rate of unmatched 446 flows that use $S_{j}$ as the ingress of egress and $S_{i}$ as the mid${ }_{447}$ dle hop. Then $\lambda_{i}^{F, j} l$ is the expected number of common flow 448 entries in $R_{j}^{t, L} \cap R_{i}^{t, F}$. During the table-overflow attack, since the 449 attacker sends lots of flows through its physically connected 450 switch $S_{j}$, these attack flows can make $C F E_{j}(t)$ increase greatly 451 and become larger comparing with the normal switches.

\section{C. Embedding the Monitoring Mechanism}

453 To make our traffic features practical and meaningful, we 454 propose a monitoring mechanism which can be embedded into 455 the original routing process of SDN. Considering the fact that 456 the control link of SDN is limited and unsuitable for heavy 457 statistics applications [35], our monitoring mechanism uti458 lizes the existing interactions between the switches and the 459 controller as shown in Fig. 4.

460 When SDN-enabled switches finish their registrations, our 461 monitoring mechanism views the established topology in the 462 controller as the graph $G=(S, L)$. Using Algorithm 1, the 463 potential targets in $G$ can be identified. During this step, 464 the controller gets the $\lambda_{i}^{F}, \lambda_{G}$ and $\lambda_{i}^{F, j}$ according to the flow 465 arrival rate $\lambda_{i, j}$ of each path and the network topology. Next, 466 for example $S_{\text {mid }}$ is a potential target and an unmatched flow ${ }_{467}$ uses $S_{\text {mid }}$ as the middle hop in its flow path, after the controller 468 assigns the flow entries to each hop, the monitoring mech469 anism updates the local flow consumptions of $S_{s r c}$ and $S_{d s t}$

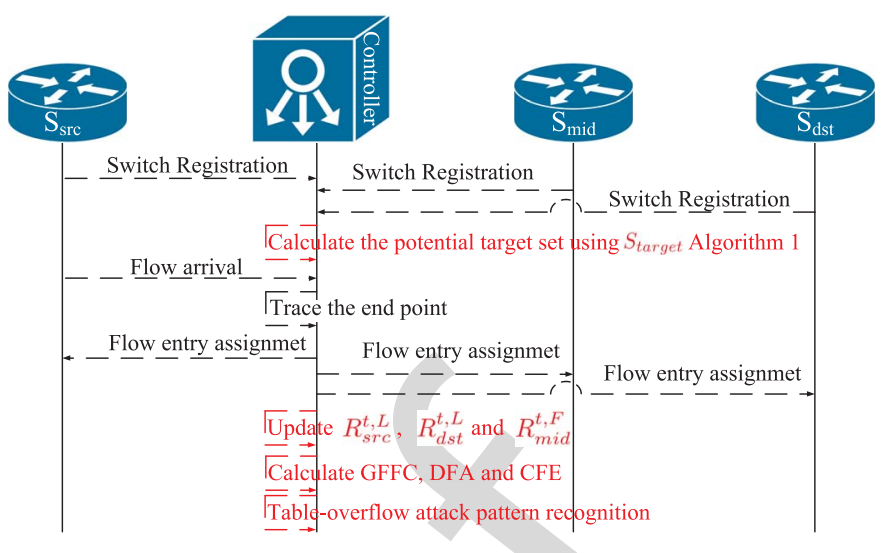

Fig. 4. Embedded monitoring steps in the SDN routing process.

and the foreign flow consumption of $S_{\text {mid }}$. Based on this, our 470 proposed traffic features can be calculated without getting data 471 plane statistics from the SDN-enabled switches. With enough 472 traffic feature samples under normal circumstances and table- 473 overflow attacks, our monitoring mechanism can recognize 474 $\begin{array}{ll}\text { such an attack and locate the attacker attached switches. } & 475\end{array}$

\section{Mitigate the TABle-Overflow Attack}

In this section, we propose a mitigation mechanism for the 477 table-overflow attack using the token bucket model. Our mit- 478 igation mechanism aims to keep the user's transmission rate 479 while limiting the attacker's transmission rate.

480

\section{A. Flow Entry Token Bucket}

481

The classic token bucket is a model used to control the ${ }_{482}$ packets' average transmission rate and burstiness in computer ${ }_{483}$ networks. In that model, the token is added into the bucket at ${ }_{484}$ a fixed rate and the bucket has finite capacity. When a packet 485 is transmitted, a token is removed from the bucket. Therefore, 486 the token add rate decides the average transmission rate of ${ }_{487}$ packets and the bucket capacity determines the burstiness of 488 the packet transmission.

Given the location of attacker by our monitoring mecha- 490 nism, we build an FETB (flow entry token bucket) for the 491 attacker attached switch to limit its transmission rate of attack 492 flows. When a flow from the attacker attached switch tries to 493 use the victim switch as the middle hop, a token is removed 494 from the FETB. To control the average transmission rate of 495 the attack flows, the fixed token add rate (denoted by TAR) is 496 defined as

$$
T A R=\lambda_{i}^{F, j}
$$

when $S_{i}$ is the victim switch and $S_{j}$ is the attacker attached ${ }_{499}$ switch.

Meanwhile, we set the bucket capacity (denoted by BC) 501 with the consideration of the current flow table vacancy on 502 the victim switch. It should be noted that, given $B C$ and $T A R, 503$ the attack time (denoted by $T$ ) and the attack rate (denoted by 504 $A R)$ satisfy that

505

$$
T=\frac{B C}{A R-T A R}, \quad A R>T A R .
$$




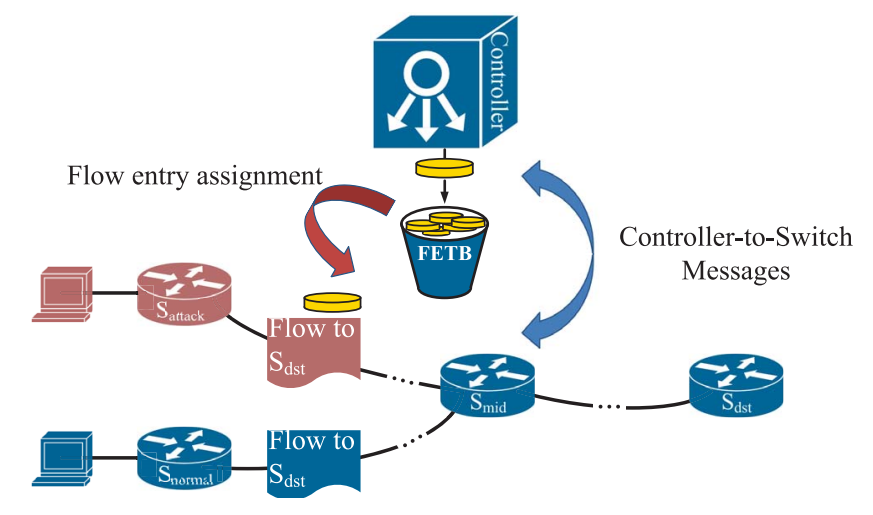

Fig. 5. The working process of the flow entry token bucket.

507 Since the attacker always sends non-repeating unmatched 508 packets, the flow entries for the attack flows are removed by 509 the idle timeout. Denoting the idle timeout value as $I T$, we 510 can calculate the maximum table consumption of the attack 511 (denoted as $M T C$ ) by

$$
512 \quad M T C=\left\{\begin{array}{c}
T \cdot A R+(I T-T) \cdot T A R=B C+I T \cdot T A R, \\
T<I T \quad\left(\Leftrightarrow A R>\frac{B C}{I T}+T A R\right) ; \\
I T \cdot A R, \\
T \geq I T\left(\Leftrightarrow \frac{B C}{I T}+T A R \geq A R>T A R\right) .
\end{array}\right.
$$

513 lue of 514 For different attack rates, we note that the maximum value of $515 M T C$ is $(B C+I T \cdot T A R)$. Since the current flow table vacancy 516 (denoted by FTV) on the victim switch can be acquired by the 517 controller using the Controller-to-Switches Messages [4], and 518 the attacker attached switch's proportion is $\frac{\lambda_{i}^{F, j}}{\lambda_{i}}$, to prevent the 519 attacker overloads the victim switch, the maximum value of 520 MTC should satisfy

521

$$
B C+I T \cdot T A R \leq F T V \frac{\lambda_{i}^{F, j}}{\lambda_{i}} .
$$

522 Therefore, the bucket capacity of the FETB is

523

$$
B C=\lambda_{i}^{F, j}\left(\frac{F T V}{\lambda_{i}}-I T\right) .
$$

\section{${ }_{524}$ B. Embedding the Mitigation Mechanism}

${ }_{525}$ To make our work practical, we embed the mitigation 526 mechanism into the original routing process of SDN shown ${ }_{527}$ Section III. As the example shown in Fig. 5, when the moni528 toring mechanism detects a table-overflow attack against $S_{\text {mid }}$ 529 and locates the attacker at $S_{\text {attack, }}$, it activates the mitigation 530 mechanism. The mitigation mechanism uses the Controller531 to-Switch Messages [4] to make sure the current FTV of $S_{\text {mid }}$. 532 Based on this, the FETB for $S_{\text {attack }}$ is established, and its $B C$ 533 and TAR can be calculated according to (15) and (11).

${ }_{534}$ After this, when a flow from $S_{\text {attack }}$ tries to use $S_{\text {mid }}$ as the 535 middle hop, $S_{\text {attack }}$ sends the routing request to controller. The 536 controller removes a token from the FETB and assigns routing 537 rules to each switch in the flow path. If there are no tokens in 538 the FETB, the flow is cached or discarded at $S_{\text {attack. Since the }}$ 539 burstiness in the FETB model is determined by $B C$, and we have set $B C$ with the consideration of the current flow table 540 vacancy, the transmission rate of attack flows can be limited 541 at $S_{\text {attack }}$. In contrast, the flow from $S_{\text {normal }}$ can be transmitted 542 without a token.

543

However, attackers may repeat their attack flows in time 544 to avoid attack flows being evicted by the idle timeout. In 545 this case, despite limiting the attack rate at $S_{\text {attack, }}$, the fixed 546 token add rate may let the attackers occupy the victim switch 547 slowly. Therefore, we use a dynamic token add rate, once the 548 number of flow entries caused by the attacker attached switch 549 exceeds their expected value $F T V \frac{\lambda_{i}^{F, j}}{\lambda_{i}}$. The token add rate is 550 determined by the rate of Flow Removed Message [4] from $S_{i}$. 551 When a flow from the attacker attached switch is finished at 552 $S_{i}$, its Flow Removed Message from $S_{i}$ makes a token added 553 into the bucket. In this way, FETB protects the switch against 554 such repeated attack flows.

\section{Simulation AND RESUlT}

In this section, we first present simulations to demonstrate 557 the performance of our monitoring mechanism in WAN, LAN 558 and data center scenarios. Then we present experiments in 559 our testbed to demonstrate the performance of our mitigation 560 mechanism for the table-overflow attack.

1) Simulation Scenarios and Key Parameters: In this paper 563 we perform our simulation over three typical types of SDN 564 application scenarios: WAN, LAN and data center. For the 565 WAN scenario, we study the recent simulation instance of soft- 566 ware defined WAN [36] and utilize the Barabasi-Albert (BA) 567 model [37] to build the network topologies with the property 568 of scale free. For the LAN scenario, we adopt the real router- 569 level dataset from CAIDA [38]. The dataset is computed by 570 the Internet Topology Data Kit (ITDK) under LAN router- 571 level topologies. For the data center, we utilize the $\operatorname{BCube}(n, k) 572$ model [39] to build the network topologies, where $n$ means the 573 port number of each switch and $k$ means the recursion times. 574 In the BCube model, both switches and servers are able to 575 forward flows while only servers are the endpoints of paths. 576 Users or attackers are only connected with or reside in servers. 577

We build different scales of topologies for each type of 578 simulation scenario. For the WAN and LAN scenarios, we 579 build the networks with the switch number $N$ varying from 100580 to 1000 . For the data center scenario, as the related study [36] 581 recommended, we apply $\operatorname{BCube}(n, k)$ model with $n$ varying 582 from 8 to 18 when $k$ is 1 and 2 . Since the number of switches 583 in the BCube topology is $n^{k} \cdot(k+1)$, the switch number $N$ of 584 the data center topology scales from 16 to 972 .

585

In the simulation topologies, as shown in the latter part 586 of this paper, the potential target switch generally holds 587 responsible for hundreds of paths. Considering the traffic char- 588 acteristics shown in the related studies [5], [40] that the hot 589 switch can receive thousands of new flows per second, we set 590 the average flow arrival rate of each path as about 10 flows per 591 second. Besides, since different path holds different amount 592 of traffic, we further set the flow arrival rate of each path 593 randomly between 1 to 20 flows per second. 


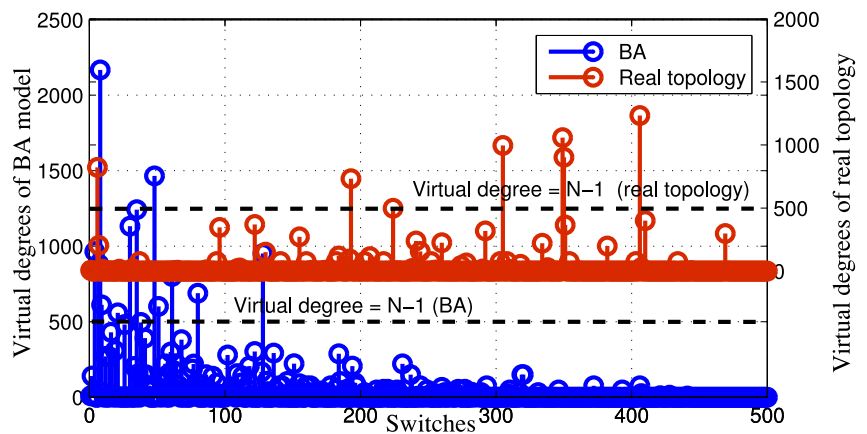

Fig. 6. The virtual degree $d_{i}$ of each switch in the BA and real router-level graphs, $N=500$.

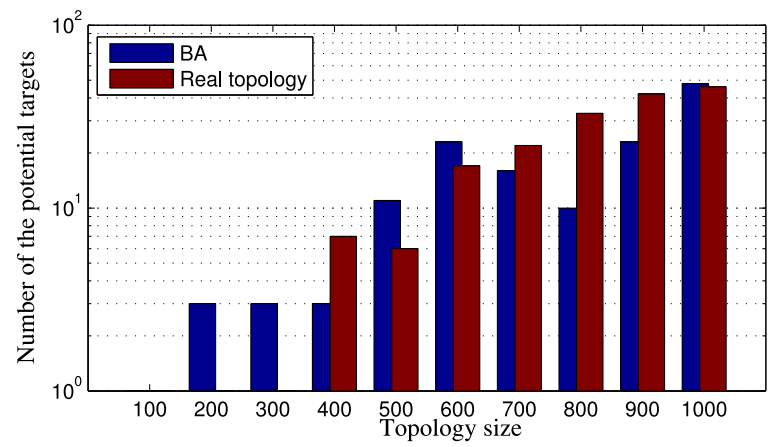

Fig. 7. From $N=100$ to $N=1000$, the number of the potential targets in the BA and real router-level graphs.

595 2) Simulation Results: As aforementioned in Algorithm 1, we 596 define the potential targets using the switches' virtual degrees. 597 Fig. 6 shows the virtual degrees of the switches in BA graph 598 and real router-lever graph when their sizes are 500. According 599 to the $d_{i}=N-1$ line, there are 12 and 6 potential targets in 600 BA graph and real router-level graph. Since the BA graph is 601 built by adding nodes to a small seed topology, the potential 602 targets of BA graph are mainly distributed in the earlier added 603 nodes. When $N$ varies from 100 to 1000 , we get the number of 604 the potential targets shown in Fig. 7. In general, the number of 605 the potential targets increases with the topology size $N$.

606 In contrast, as shown in Fig. 8, the switches in the BCube 607 graph have the same virtual degree, which is decided by the 608 parameter $n$ and $k$ and satisfies $d_{i}=\left(\begin{array}{l}n \\ 2\end{array}\right) \cdot n^{k}$, the detail expla609 nation can be found in the Appendix. With the increasing of $610 \mathrm{n}$, the virtual degree of switch increases exponentially and it 611 deviates from the $d_{i}=N-1$ line obviously.

612 From Fig. 8, we can get some further results. First, due to the 613 dense placement of servers $\left(n^{(k+1)}\right)$ and the sparse placement of 614 switches $\left(n^{k} \cdot(k+1)\right)$, switches in the BCube-based data center 615 face much more forwarding task comparing with the ones in 616 the WAN and LAN scenarios, and they are more likely to be 617 compromised by the table-overflow attack. Second, the heavy 618 forwarding task of switches in $\operatorname{BCube}(n, k)$ model (each switch 619 maintains $\left(\begin{array}{l}n \\ 2\end{array}\right) \cdot n^{k}$ paths) makes $d_{i}>N-1$ a loose definition 620 for potential target. However, since the $\operatorname{BCube}(n, k)$ topology 621 is homogeneous (each switch has the same virtual degree), our 622 definition is suitable. Last, the recursive property of BCube 623 makes switches homogeneous in the topology, therefore the

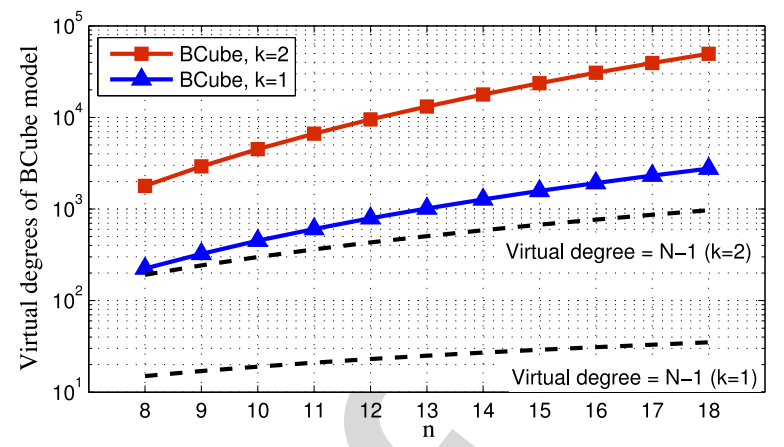

Fig. 8. The virtual degree $d_{i}$ of switches in the BCube graphs, the virtual degree $d_{i}$ of switches in the $\operatorname{BCube}(n, k)$ graph satisfies that $d_{i}=\left(\begin{array}{c}n \\ 2\end{array}\right) \cdot n^{k}$.

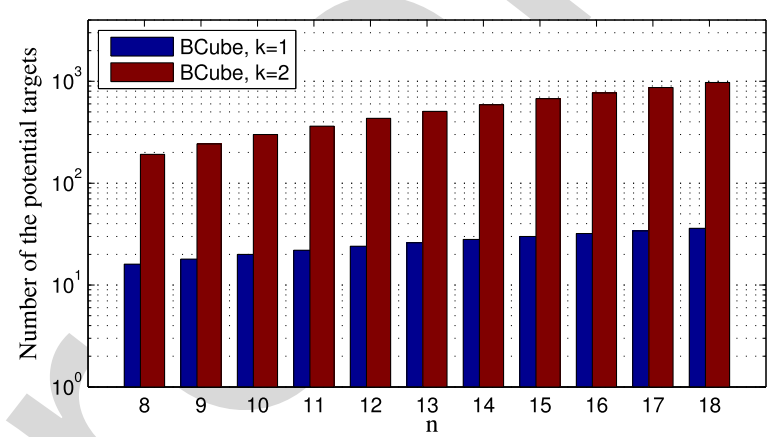

Fig. 9. From $n=8$ to $n=18$, the number of the potential targets in the $\operatorname{BCube}(n, k)$ graphs.

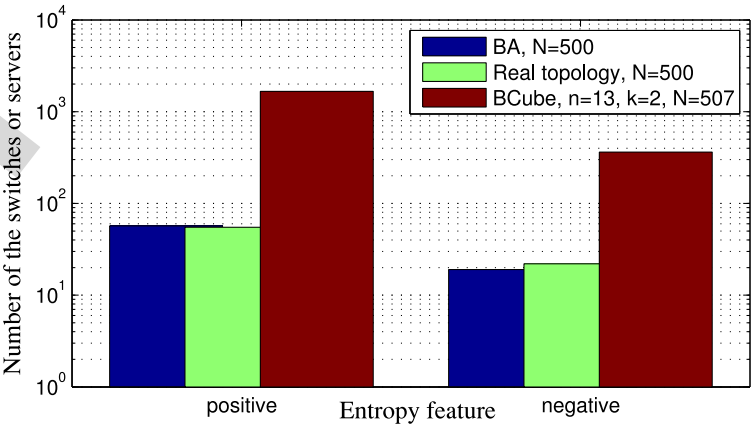

Fig. 10. For a certain target, the switch or server numbers of different entropy features.

number of potential targets in BCube graph increases linearly 624 with the growth of topology size as shown in Fig. 9.

To evaluate the performance of our monitoring mechanism, 626 we first compare our DFA with the entropy-based traffic fea- 627 ture. It should be noted that, the accuracy of the entropy-based ${ }_{628}$ traffic feature depends on the randomness of the flows [18]. 629 However, switches (or servers in the BCube graph) have dif- 630 ferent numbers of paths which use the target as the middle 631 hop. This affects the randomness of the flows. For a certain 632 potential target, Fig. 10 shows the switch or server numbers of 633 different entropy features. The "positive" switches or servers 634 have fewer paths while the "negative" switches have more 635 paths than the average value. When the attackers send flows 636 through the "positive" devices, the entropy features of the 637 target increase. When the attackers send flows through the 638 "negative" devices, the entropy features of the target decrease. 639 


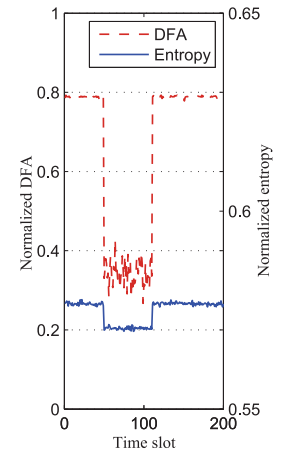

(a)

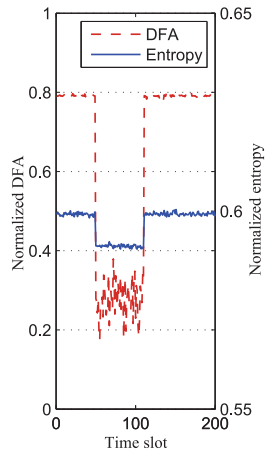

(b)

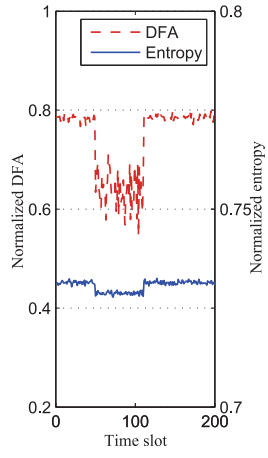

(c)
Fig. 11. DFA and the Src-IP entropy values, attacker attached switches or servers are "negative" devices, $l=10$ seconds, 10 attackers, the attack rate is 500 flows/s, (a) BA model, $N=500$, (b) real router-level, $N=500$, (C) BCube model, $N=507$.

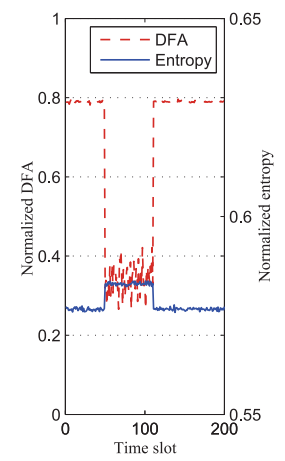

(a)

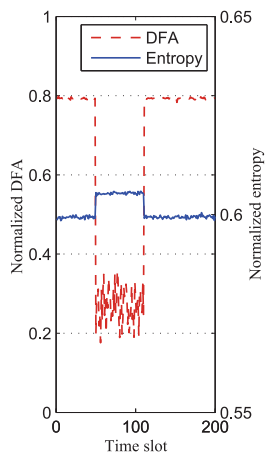

(b)

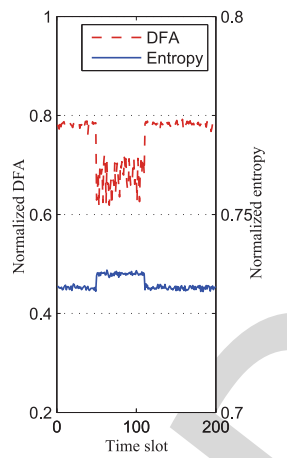

(c)
Fig. 12. DFA and the Src-IP entropy values, attacker attached switches or servers are "positive" devices, $l=10$ seconds, 10 attackers, the attack rate is 500 flows/s, (a) BA model, $N=500$, (b) real router-level, $N=500$, (C) BCube model, $N=507$.

640 Using the normalized DFA and normalized Src-Ip entropy, ${ }_{641}$ Fig. 11 shows the monitoring results when attacker attached 642 switches or servers are "negative" devices. We can see that, 643 both features can differentiate the table-overflow attack, and ${ }_{644}$ DFA shows a better sensitivity.

645 However, when attacker attached switches or servers are ${ }_{646}$ "positive" devices, the entropy-based method is not feasible. 647 Fig. 12 shows the monitoring results when attacker attached 648 switches or servers are "positive" devices. We can see that, the ${ }_{649}$ Src-Ip entropy value increases and leads to a False Negative 650 (FN) error while DFA feature keeps a well sensitivity to the 651 attack.

652 Then we compare our GFFC with the average attack rate of 653 attackers in Fig. 13. As discussed in Section V, the attackers 654 can hide themselves by splitting the attack cost. We can see 655 that, with the growth of attack numbers, it becomes harder to 656 identify an attack according to their request rate. To launch the 657500 flows/s table-overflow attack, the average attack cost of the 65815 attackers is only less than 35 flows/s, which can be mistaken 659 for normal traffic fluctuations. However, the attack payment ${ }_{660}$ CFFC is noteworthy. As shown in Fig. 13, it grows linearly 661 with the attack rate and is independent with the number of 662 attackers.

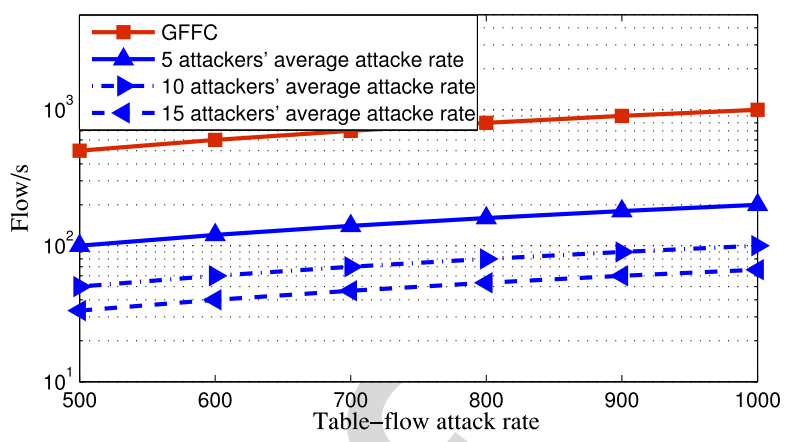

Fig. 13. GFFC and the average attack rate of attackers.

TABLE III

Key PARAMETERS OF OUR EXPERIMENTS

\begin{tabular}{l|l}
\hline \hline parameter & Value \\
\hline maximum allowed $M T C$ & 1500 flow entries \\
$T A R$ & $50 / \mathrm{s}$ \\
$I T$ & $10 \mathrm{~s}$ \\
$A R$ & 100 flow/s; 150 flow/s; 200 flow/s \\
\hline
\end{tabular}

Using DFA and GFFC, we monitor the table-overflow attack 663 in different types and scales of topologies, and we summarize 664 the results in Fig. 14. According to the Receiver Operating 665 Characteristic (ROC) curves and the Confusion Matrixes, we ${ }_{666}$ can see that, our method achieves $100 \%$ accuracy with a 667 False Positive (FP) rate ranging from 6.3\% to $13.7 \%$. During 668 the simulations, as depicted by Fig. 14(b), Fig. 14(d) and 669 Fig. 14(f), our method archives an accuracy of $96.4 \%$ in the 670 BA graphs, an accuracy of $95.1 \%$ in the real router-level 671 graphs and an accuracy of $93.4 \%$ in the BCube graphs. 672

To further locate the attacker attached switches, we calculate 673 $C F E$ when the table-overflow attack is identified. For BA and 674 real topologies, we get the $C F E$ values shown in Fig. 15. The 675 $C F E$ values of the normal switches range in $(0.8,1.2)$ while the 676 attack attached switches range in $(1.5,1.9)$. For the BCube, 677 as shown in Fig. 16, the $C F E$ values of the normal servers 678 range in $(0.7,1.4)$ while the attack attached switches range in 679 $(1.8,2.0)$.

\section{B. Evaluating the Mitigation Mechanism}

681

1) Simulation Settings: To test the performance of our mit- 682 igation mechanism, we program the flow entry token bucket 683 as an application in OpenDaylight controller [41] using REST 684 API. We use OpenvSwitch [33] as the SDN-enabled switch. 685 The controller and each SDN-enabled switch are equipped 686 with an Intel Xeon E5606 CPU working at $2.13 \mathrm{GHz}$ and an ${ }_{687}$ 8G memory chip. They are operated with Ubuntu Linux 12.04. 688

We use two laptops to send and accumulate flow entries on 689 the middle hop switch. The attack flows are generated in the 690 form of the ICMP ping flood using Hping [42]. The parameters 691 that we used are listed in Table III.

692

2) Simulation Results: To avoid the table overflow on the 693 victim switch, we need to control the bucket capacity with 694 the consideration of the current flow table vacancy. According 695 to the experiment settings and the analysis of (15), the cal- 696 culation result of the maximum $B C$ is 1000 . Based on this, 697 the controller can limit the transmission rate of attack flows. 698 


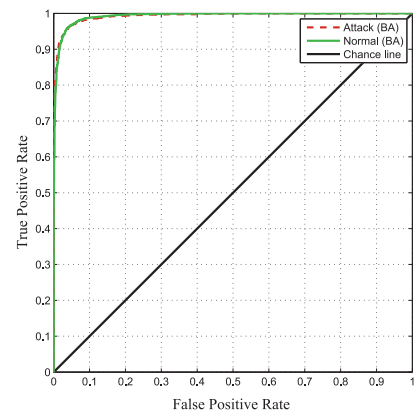

(a)

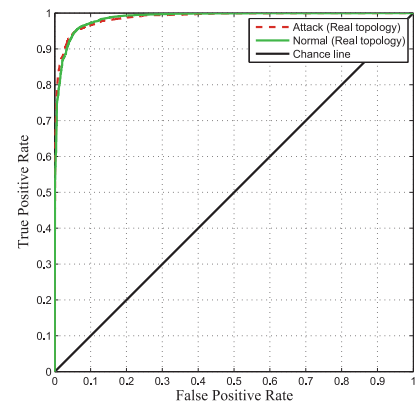

(c)

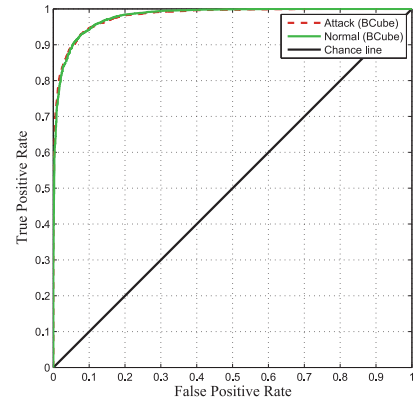

(e)

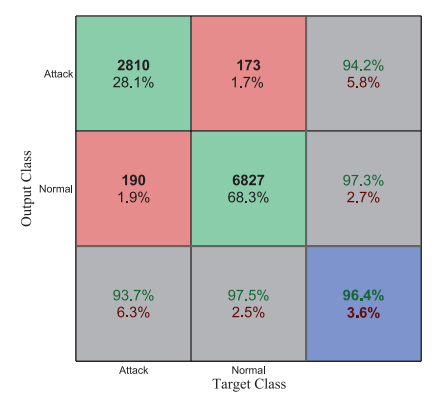

(b)

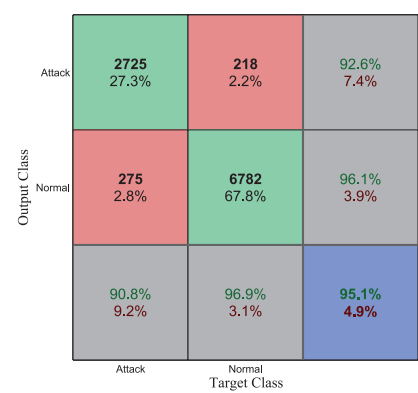

(d)

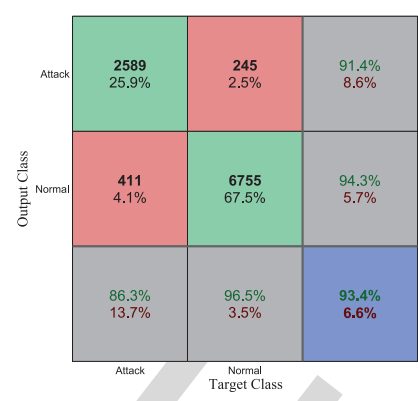

(f)
Fig. 14. Monitoring performances, (a), (c) and (e) are ROCs of BA model, real router-level and BCube model, (b) (d) and (f) are confusion matrixes of BA model, real router-level and BCube model.
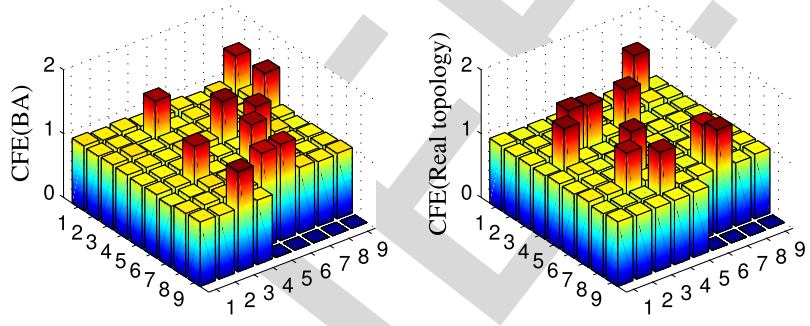

Fig. 15. $\quad N=500,10$ attackers, for a certain target, the $C F E$ values of switches in the BA and real router-level graphs.

699 For different attack rates, we get the rate limiting results in 700 Fig. 17. As can be seen, when the bucket has no tokens, the 701 attack flows' transmission rate is limited by the fixed token 702 add rate. The higher the attack rate is, the earlier the limiting 703 action comes. Over a long run, the number of the attack flows 704 is limited to $B C+t * T A R$.

705 Since the MTC indicates the maximum allowed table 706 consumption for the attackers, we record the flow table

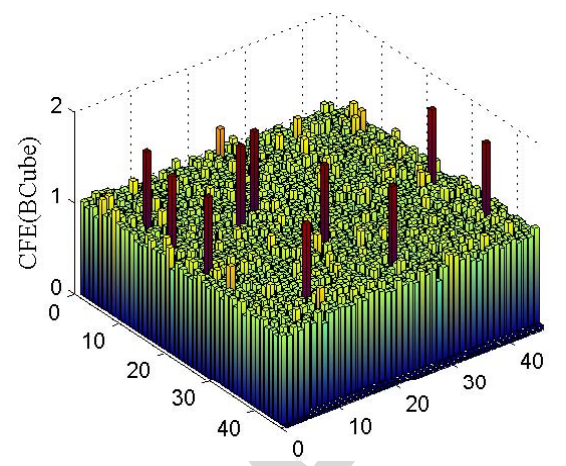

Fig. 16. $n=13, k=2,10$ attackers, for a certain target, the $C F E$ values of servers in the BCube graph.

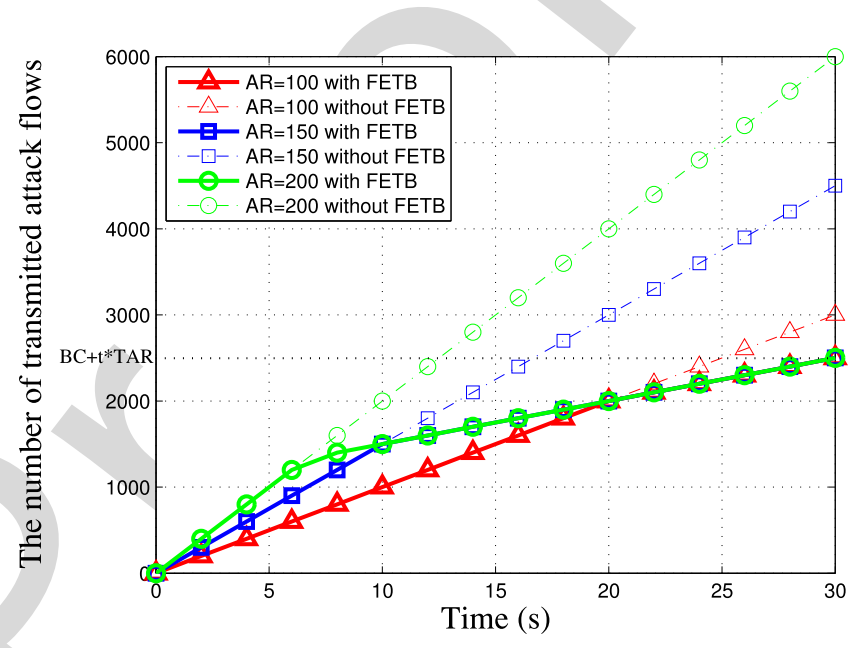

Fig. 17. The effect of FETB on the attacker attached switch.

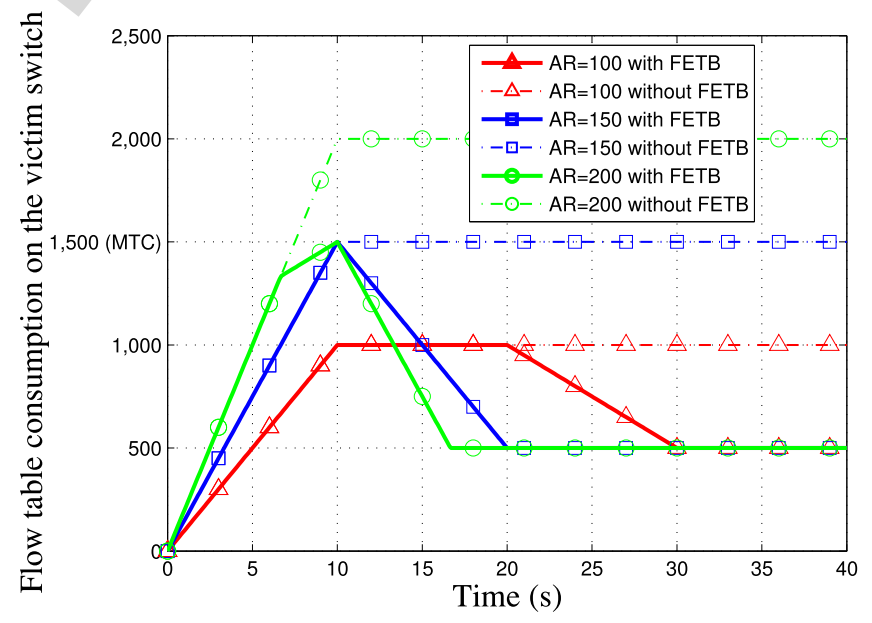

Fig. 18. The effect of FETB on the victim switch.

consumptions of the attackers and summarize them in Fig. 18. 707 We can see that, with the rate limiting and the calculated 708 bucket capacity, the maximum flow table consumption of the 709 attackers is limited to MTC. Over a long run, the flow table 710 consumption of the attackers is $I T * T A R$.

However, attackers may repeat their attack flows in time 712 to avoid attack flows being evicted by the idle timeout. To 713 simulate the effect of repeated attack flows, we force the con- 714 troller to assign static flow entries for the non-repeat attack 715 


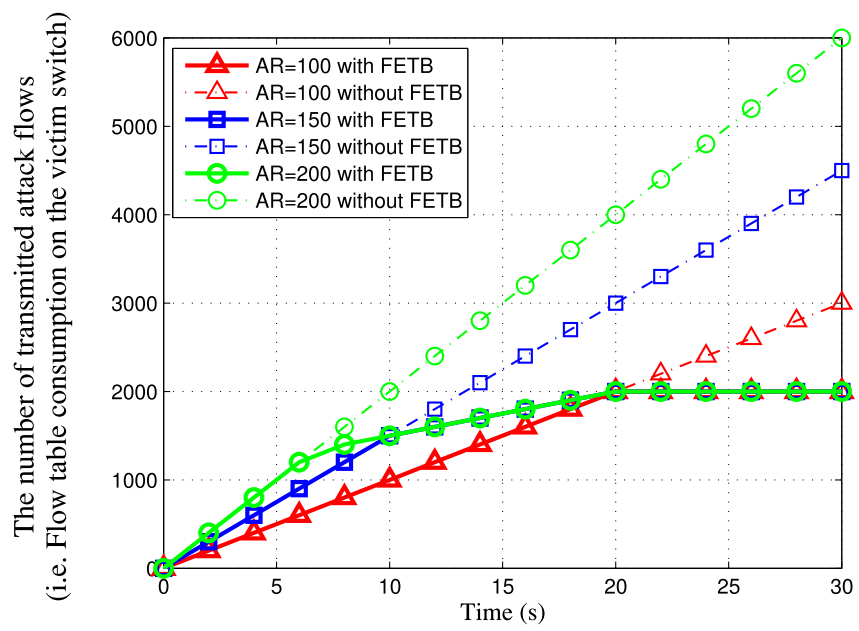

Fig. 19. The effect of FETB on both the attacker attached switch and the victim switch.

716 flows. The static flow entries have infinite idle timeout value, 717 and they will not be removed until we delete them manually. 718 In this case, the performance of FETB is shown in Fig. 19. ${ }_{719}$ We use the dynamic token add rate, once the number of flow 720 entries caused by attack flows exceeds 2,000.

721 Comparing with the recent QoS-aware mitigation strat722 egy [32], which redirects attack flows to the idle peer switch, 723 our mitigation mechanism limits the attack rate at the attacker 724 attached switches. Using the proper bucket capacity and 725 token add rate, FETB avoids the table overflow on the 726 victim switch.

727

\section{CONCLUSION}

${ }_{728}$ In this paper, we examined the table-overflow attack in 729 SDN with practical considerations and concluded that exist730 ing solutions were unable to protect the system under such 731 an attack. To address this issue, we first proposed a monitor732 ing mechanism to detect the table-overflow attack during the 733 original routing process of SDN. Three traffic features were 734 designed to differentiate the attack and locate the attackers. 735 We then proposed a mitigation mechanism to reduce the trans736 mission rate of attack flows on the attacker attached switch. ${ }_{737}$ We designed a flow entry token bucket model to avoid the 738 table overflow on the victim switch. Extensive simulations 739 were conducted to evaluate and show the effectiveness of our 740 solution against the table-overflow attack.

741

742 The $\operatorname{BCube}(n, k)$ model creates iterative graphs, the small743 est iteration unit is $\mathrm{BCube}_{0}$ and the largest iteration unit is 744 BCube $_{k-1}$. For example, Fig. 20(a) shows the topology of 745 BCube $(4,1)$. In fact, in such an iterative graph, the switches 746 in different levels are homogenous and can be exchanged. ${ }_{747}$ When we exchange the level 0 switches with the level 1 748 switches in Fig. 20(a), we get Fig. 20(b). The iterative feature 749 of $\operatorname{BCube}(n, k)$ model makes the switches have the same $d_{i}$. 750 Since the switches have the same $d_{i}$, we only need to find 751 one switch's $d_{i}$. The simplest way is to choose a switch in

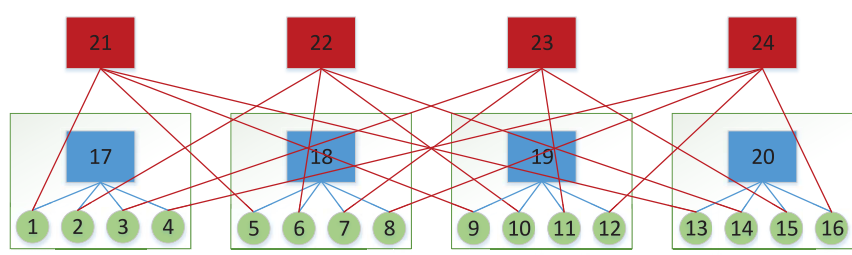

(a)

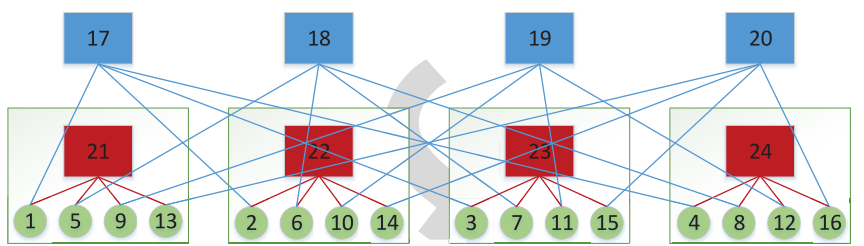

(b)

Fig. 20. The topology of BCube(4,1), the rectangles represent switches and the circles represent servers, the switches have the same $d_{i}=24$.

the highest level $k$, since only flows between two BCube ${ }_{k-1} \mathrm{~S} 752$ use it as the middle hop. Then according to the number of 753 severs in each BCube $_{k-1}$ (i.e., $n^{k}$ ) and the number of possible 754 combinations of two BCube $k_{k-1}$ s (i.e., $\left(\begin{array}{l}n \\ 2\end{array}\right)$ ), we get the $d_{i}$ of 755 the level $k$ switch as $d_{i}=\left(\begin{array}{l}n \\ 2\end{array}\right) \cdot n^{k}$.

\section{REFERENCES}

[1] F. Hu, Q. Hao, and K. Bao, "A survey on software-defined network and 758 OpenFlow: From concept to implementation," IEEE Commun. Surveys 759 Tuts., vol. 16, no. 4, pp. 2181-2206, 4th Quart., 2014.

[2] B. A. A. Nunes, M. Mendonca, X.-N. Nguyen, K. Obraczka, and 761 T. Turletti, "A survey of software-defined networking: Past, present, 762 and future of programmable networks," IEEE Commun. Surveys Tuts., 763 vol. 16, no. 3, pp. 1617-1634, 3rd Quart., 2014.

[3] M. McKeown et al., "OpenFlow: Enabling innovation in campus 765 networks," ACM SIGCOMM Comput. Commun. Rev., vol. 38, no. 2, 766 pp. 69-74, 2008.

[4] (2014). OpenFlow Switch Specification. [Online]. Available: 768 http://www.opennetworking.org/

[5] K. Kalapriya and S. Banerjee, "Compact TCAM: Flow entry compaction 770 in TCAM for power aware SDN," in Proc. 14th Int. Conf. Distrib. 771 Comput. Netw., Mumbai, India, 2013, pp. 439-444. 772

[6] D. Kreutz et al., "Software-defined networking: A comprehensive 773 survey," Proc. IEEE, vol. 103, no. 1, pp. 14-76, Jan. 2015.

[7] P. Zhang, H. Wang, C. Hu, and C. Lin, "On denial of service attacks 775 in software defined networks," IEEE Netw., vol. 30, no. 6, pp. 28-33, 776 Nov./Dec. 2016.

[8] (2016). Noviswitch. [Online]. Available: http://noviflow.com/products/ 778 noviswitch/

[9] Y. Kanizo, D. Hay, and I. Keslassy, "Palette: Distributing tables in 780 software-defined networks," in Proc. IEEE INFOCOM, Turin, Italy, 781 2013, pp. 545-549.

[10] N. Kang, Z. Liu, J. Rexford, and D. Walker, "Optimizing the "one 783 big switch' abstraction in software-defined networks," in Proc. ACM 784 CoNEXT, Santa Barbara, CA, USA, 2013, pp. 12-24.

[11] B. Leng, L. Huang, X. Wang, H. Xu, and Y. Zhang, "A mechanism for 786 reducing flow tables in software defined network," in Proc. IEEE ICC, 787 London, U.K., 2015, pp. 5302-5307.

[12] L. Zhang, S. Wang, S. Xu, R. Lin, and H. Yu, "TimeoutX: An adaptive 789 flow table management method in software defined networks," in Proc. 790 IEEE GLOBECOM, San Diego, CA, USA, 2015, pp. 1-6. 791

[13] D. B. Rawat and S. R. Reddy, "Software defined networking architecture, 792 security and energy efficiency: A survey," IEEE Commun. Surveys Tuts., 793 vol. 19, no. 1, pp. 325-346, 1st Quart., 2017.

[14] S. Scott-Hayward, S. Natarajan, and S. Sezer, "A survey of security in 795 software defined networks," IEEE Commun. Surveys Tuts., vol. 18, no. 1, 796 pp. 623-654, 1st Quart., 2016.

[15] I. Ahmad, S. Namal, M. Ylianttila, and A. Gurtov, "Security in software 798 defined networks: A survey," IEEE Commun. Surveys Tuts., vol. 17, 799 no. 4, pp. 2317-2346, 4th Quart., 2015. 
801 [16] R. Kandoi and M. Antikainen, "Denial-of-service attacks in OpenFlow 802 SDN networks," in Proc. IFIP/IEEE Int. Symp. Integr. Netw. Manag., 803 Ottawa, ON, Canada, 2015, pp. 1322-1326.

804 [17] H. T. N. Tri and K. Kim, "Assessing the impact of resource attack 805

812 [20] (2015). Pica8. [Online]. Available: http://www.pica8.com/

813 [21] A. R. Curtis et al., "DevoFlow: Scaling flow management for highEmerg. Topics Comput, vol. 4, no. 2, pp. 252-261, Apr./Jun. 2015.

A. Vishnoi, R. Poddar, V. Mann, and S. Bhattacharya, "Effective switch memory management in OpenFlow networks," in Proc. 8th ACM Int. Conf. Distrib. Event Based Syst., Mumbai, India, 2014, pp. 177-188.

27] H. Huang, S. Guo, P. Li, W. Liang, and A. Y. Zomaya, "Cost minimization for rule caching in software defined networking," IEEE Trans. Parallel Distrib. Syst., vol. 27, no. 4, pp. 1007-1016, Apr. 2016.

[28] H. Li, S. Guo, C. Wu, and J. Li, "FDRC: Flow-driven rule caching optimization in software defined network," in Proc. IEEE ICC, London, U.K., 2015, pp. 5777-5782.

[29] R. Doriguzzi-Corin, D. Siracusa, E. Salvadori, and A. Schwabe, "Empowering network operating systems with memory management techniques," in Proc. IEEE/IFIP Netw. Oper. Manag. Symp., Istanbul, Turkey, 2016, pp. 740-744.

[30] S. Shin, V. Yegneswaran, P. Porras, and G. Gu, “Avant-guard: Scalable and vigilant switch flow management in software-defined networks," in Proc. 20th ACM Conf. Comput. Commun. Security, Berlin, Germany, 2013, pp. 413-424.

31] P. Dong, X. Du, H. Zhang, and T. Xu, "A detection method for a novel DDoS attack against SDN controllers by vast new lowtraffic flows," in Proc. IEEE ICC, Kuala Lumpur, Malaysia, 2016, pp. 1-6.

32] B. Yuan et al., "Defending against flow table overloading attack in software-defined networks," IEEE Trans. Services Comput., to be published.

33] (2015). OpenvSwitch. [Online]. Available: http://openvswitch.org/

34] E.-D. Kim, S.-I. Lee, Y. Choi, M.-K. Shin, and H.-J. Kim, "A flow entry management scheme for reducing controller overhead," in Proc. 16th ICACT, 2014, pp. 754-757. ment," in Proc. 2nd ACM SIGCOMM Workshop Hot Topics Softw. Defined Netw., Hong Kong, 2013, pp. 151-152.

36] R. Cohen, L. Lewin-Eytan, J. S. Naor, and D. Raz, "On the effect of forwarding table size on SDN network utilization," in Proc. IEEE INFOCOM, Toronto, ON, Canada, 2014, pp. 1734-1742.

37] R. Albert and A.-L. Barabasi, "Statistical mechanics of complex networks," Rev. Modern Phys., vol. 74, pp. 47-97, Jan. 2002.

38] (2016). Macroscopic Internet Topology Data Kit (ITDK). [Online]. Available: http://www.caida.org/data/internet-topology-data-kit/

39] C. Guo et al., "BCube: A high performance, server-centric network architecture for modular data centers," in Proc. ACM SIGCOMM Conf. Data Commun., Barcelona, Spain, 2009, pp. 63-74.

40] (2016). The CAIDA UCSD Statistical Information for the CAIDA Anonymized Internet Traces. [Online]. Available: http://www.caida.org/data/passive/passive_trace_statistics.xml

11] OpenDaylight. (2013). A Linux Foundation Collaborative Project. [Online]. Available: http://www.opendaylight.org/

[42] (2014). Hping. [Online]. Available: http://www.hping.org/

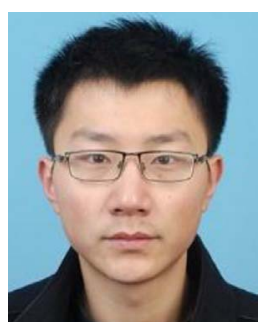

Tong Xu received the B.S. degree from Beijing 878 Jiaotong University in 2012, where he is cur- 879 rently pursuing the Ph.D. degree with the School 880 of Electronic and Information Engineering. His 881 research interests are Internet of Things, com- 882 munication system security, and software defined 883 networking.

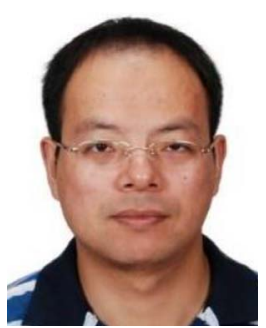

Deyun Gao received the B.Eng. and M.Eng. degrees 885 in electrical engineering and the $\mathrm{Ph} . \mathrm{D}$. degree in 886 computer science from Tianjin University, China, in 887 1994, 1999, and 2002, respectively. He spent one 888 year as a Research Associate with the Department 889 of Electrical and Electronic Engineering, Hong Kong 890 University of Science and Technology. He then spent 891 three years as a Research Fellow with the School 892 of Computer Engineering, Nanyang Technological 893 University, Singapore. In 2007, he joined the fac- 894 ulty of Beijing Jiaotong University as an Associate 895 Professor with the School of Electronics and Information Engineering and 896 was promoted to a Full Professor in 2012. In 2014, he was a Visiting Scholar 897 with the University of California at Berkeley, USA. His research interests 898 are in the area of Internet of Things, vehicular networks, and next-generation 899 Internet.

5] K. Benton, L. J. Camp, and C. Small, "OpenFlow vulnerability assess-

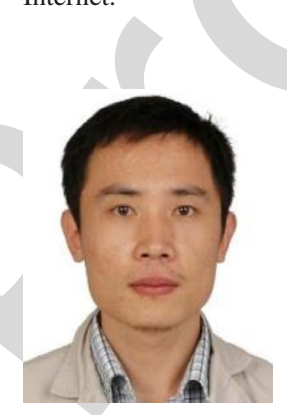

Ping Dong received the Ph.D. degree in communica- 901 tions and information system from Beijing Jiaotong 902 University, Beijing, China, in 2009. In 2015, he 903 was a Visiting Scholar with Temple University, 904 Philadelphia, PA, USA. He is currently an Associate 905 Professor with Beijing Jiaotong University. His 906 research interests include mobile Internet, software- 907 defined networking, and network security. He has 908 published over 40 research papers in the above areas. 909

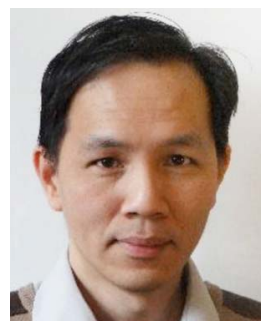

Chuan Heng Foh (SM'09) received the M.Sc. 910 degree from Monash University, Australia, in 911 1999 and the Ph.D. degree from the University 912 of Melbourne, Australia, in 2002. He spent six 913 months as a Lecturer with Monash University. In 914 2002, he joined Nanyang Technological University, 915 Singapore, as an Assistant Professor until 2012. He 916 is currently a Senior Lecturer with the University 917 of Surrey. He has authored or co-authored over 100918 refereed papers in international journals and confer- 919 ences. His research interests include protocol design 920 and performance analysis of various computer networks including wireless 921 local area and mesh networks, mobile ad hoc and sensor networks, Internet of 922 Things, 5G networks, and data center networks. He is currently an Associate 923 Editor of the IEEE ACCESS, the IEEE WIRELESS COMMUNICATIONS, and 924 the International Journal of Communications Systems. He is the Vice-Chair 925 (Europe/Africa) of the IEEE Technical Committee on Green Communications 926 and Computing.

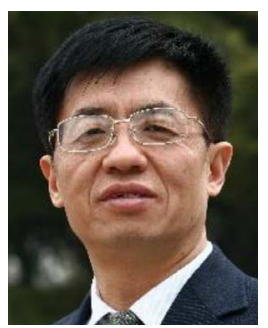

Hongke Zhang received the Ph.D. degree from the 928 University of Electronic Science and Technology 929 of China, Chengdu, China, in 1992. He is cur- 930 rently a Professor with the School of Electronic 931 and Information Engineering, Beijing Jiaotong 932 University, Beijing, China, where he currently 933 directs the National Engineering Laboratory on 934 Internet Technology for Next Generation Internet in 935 China. His research has resulted in many research 936 papers, books, patents, systems, and equipment 937 in the areas of communications and computer 938 networks. He has served on the editorial boards of several international 939 journals. 